\title{
Skipping Breakfast is Associated with Constipation in Post-Adolescent Female College Students in Japan
}

\author{
Tomoko Fujiwara \\ Faculty of Home Economics, Ashiya College, Ashiya, \\ Japan
}

\section{Introduction}

Intake of food is one of the most effective stimulations that induce bowel movement. Food intake stimulates bowel peristalsis mainly through automatic nerve system (Nobel et al., 2009). On the other hand, it is widely accepted that physiological and/or psychological stresses can induce constipation in women by suppressing the function of parasympathetic nerve networks (Whitehead, 1996).

In general, the dominance of the parasympathetic nerve system while sleeping promotes digestive and absorptive function. In this regard, the time dinner is eaten may be a factor that influences the conditions of bowel movement the following day. In addition, intake of breakfast is an important event that gives an opportunity to empty the bowels in the morning when the work day usually starts (Cummings JH). Accordingly, it is speculated that late intake of dinner or skipping breakfast can disrupt the rhythms of bowel movement, inducing various problems such as constipation.

To support the above speculation, Kunimoto et al. reported that skipping breakfast is strongly related to constipation in Japanese working women and signs of this relationship have already appeared in adolescents (Kunimoto et al., 1998). In accordance with this study, we also observed the positive relationship between breakfast skipping and constipation in Japanese young female students (Fujiwara and Nakata, 2010).

In this study, we re-evaluated the relationship between skipping breakfast and bowel movement by conducting an 11-year questionnaire survey (from 2000 to 2010) of 1,877 female college students aged between 18 and 20 years old. We further examined the relationship between dinner time and bowel movement by conducting a single year questionnaire survey in 2010.

\section{Methods}

\subsection{Respondents to a questionnaire}

The subjects were yong Japanese women aged from 18 to 20 years old who studied at the Faculty of Home Economics of Ashiya College and Kyoto Bunkyo Junior College. The study 
protocol was approved by the Committee on Food Culture at Ashiya College. We sent questionnaires to all students who belonged to the Faculty of Home Economics and Child Education between 2000 and 2010. Information regarding the aim of this study was sent with the questionnaire, and consent was obtained from all participants. The total number of the participants in the sequential studies was 1898 and we obtained responses that were suitable for statistical analysis from 1,877 students in the sequential study.

\subsection{Questionnaire items}

\subsubsection{Skipping breakfast}

All study participants completed a food-frequency questionnaire about breakfast (food intake until 9:00 am) and were divided into three groups as follows: Group I, having breakfast every morning; Group II, having breakfast one to six times a week; and Group III, having breakfast less than once a week.

\subsubsection{Body mass index (BMI) assessment}

Information on body mass (kilograms) and height (meters) of all participants were obtained from a physical examination organized by the Health Center at Ashiya College and Kyoto Bunkyo Junior College. BMI was calculated using the formula: body weight in kilograms divided by height in meters squared.

\subsubsection{Bowel movement}

The frequency of bowel movement was classified into Grade 1 (no more than once a week), Grade 2 (2-6 times a week), and Grade 3 (every day).

\subsubsection{Time of dinner intake}

Participants in 2010 were divided into three groups according to the time of dinner as follows: Group A, having dinner before 19:00; Group B, having dinner between 19:00 and 21:00; Group C, having dinner after 21:00.

\subsection{Statistical analysis}

The data are shown as mean \pm standard deviation (SD). Differences in the bowel movement among Groups I-III and A-C were analyzed by the Kruskal-Wallis test, followed by the Mann-Whitney test for multiple comparisons. The relationship between BMI and skipping breakfast was analyzed by one-way analysis of variance, followed by Scheffe's F test for multiple comparisons. $P$-values less than 0.05 were considered significant.

\section{Results}

\subsection{Changes over time in the proportions of the population showing various breakfast habits}

Among the participants, 1877 students were classified in Groups I, II and III. Annual population rates for breakfast habits between 2000 and 2010 are shown in Figure 1. Population of breakfast skipping has been within 5-22\% throughout the study. 


\subsection{Changes over time in bowel movement scores}

There was a tendency for bowel movement scores in Group I to be higher than those in the other groups throughout the study (Figure 1).

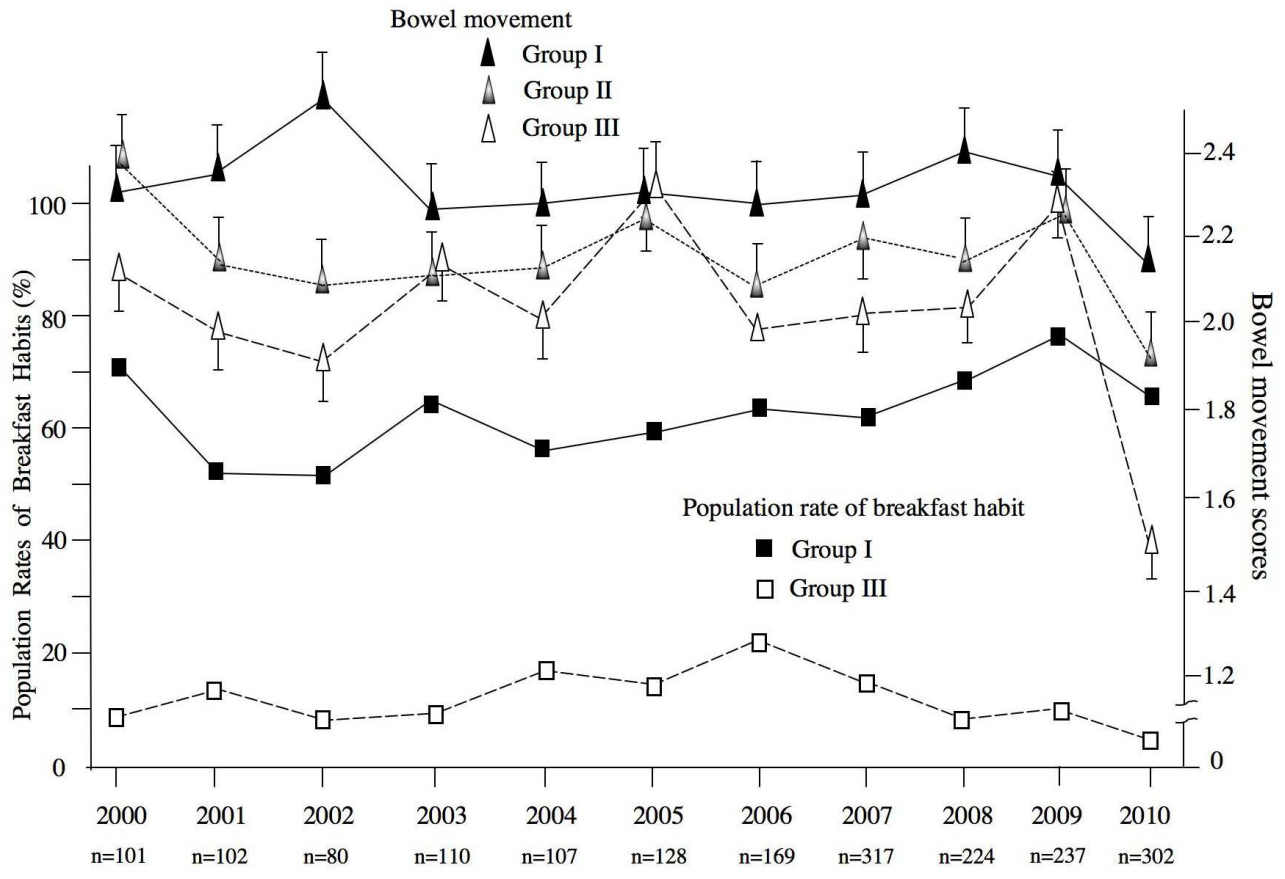

Fig. 1. Changes in population rates and bowel movement scores in Groups I, II and III throughout an 11-year surveillance period from 2000 to 2010. Population of breakfast skipping has been within 5-22\% and bowel movement scores in Group I are higher than those in the other groups throughout the study. 


\subsection{Relationship between breakfast habits and bowel movement scores}

The bowel movement score was significantly lower in Group III than in Group I and II (Figure 2).

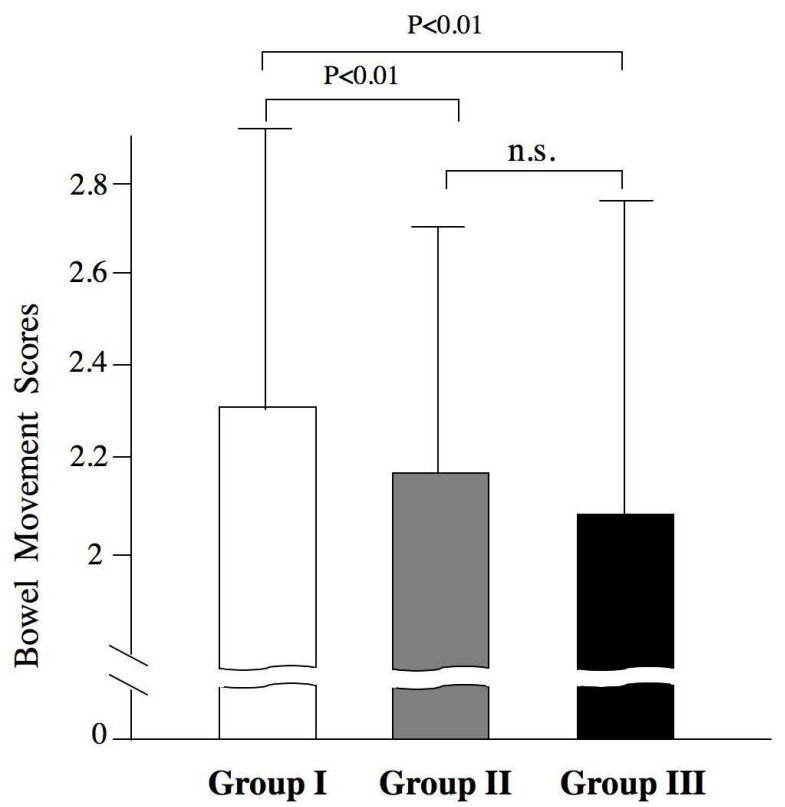

Fig. 2. Relationship between skipping breakfast and bowel movement scores. The bowel movement score was significantly lower in Group III than in Group I and Group II, suggesting that skipping breakfast induced constipation in young students. 


\subsection{The differences in BMI scores}

As shown in Figure 3, there were no significant differences in BMI scores among Group I, II and III. There were no significant changes in BMI scores throughout the observation periods.

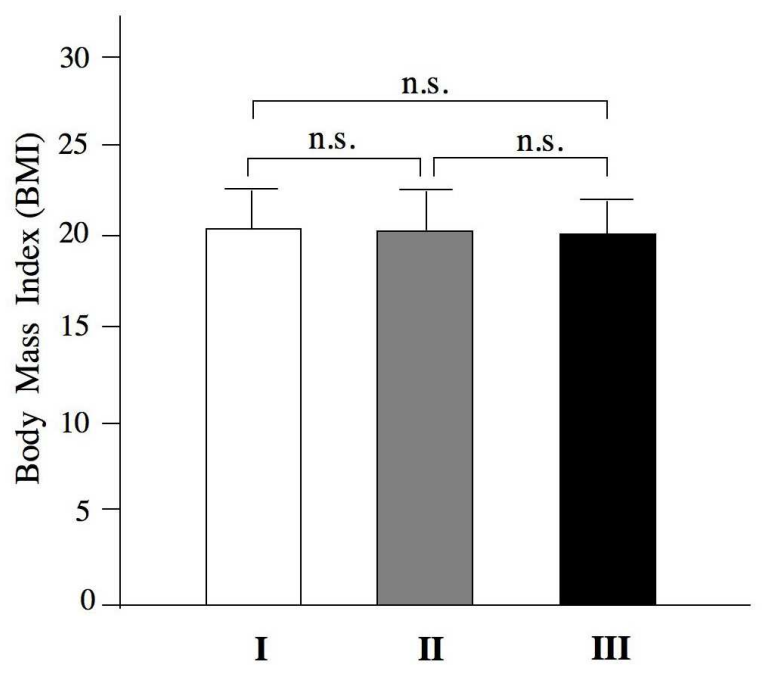

Fig. 3. Relationship between skipping breakfast and BMI. There were no significant changes in BMI scores among three groups. 


\subsection{Relationship between dinner time and bowel movement scores}

Bowel movement scores in Group A were significantly higher than those of the other two groups (Figure 4).

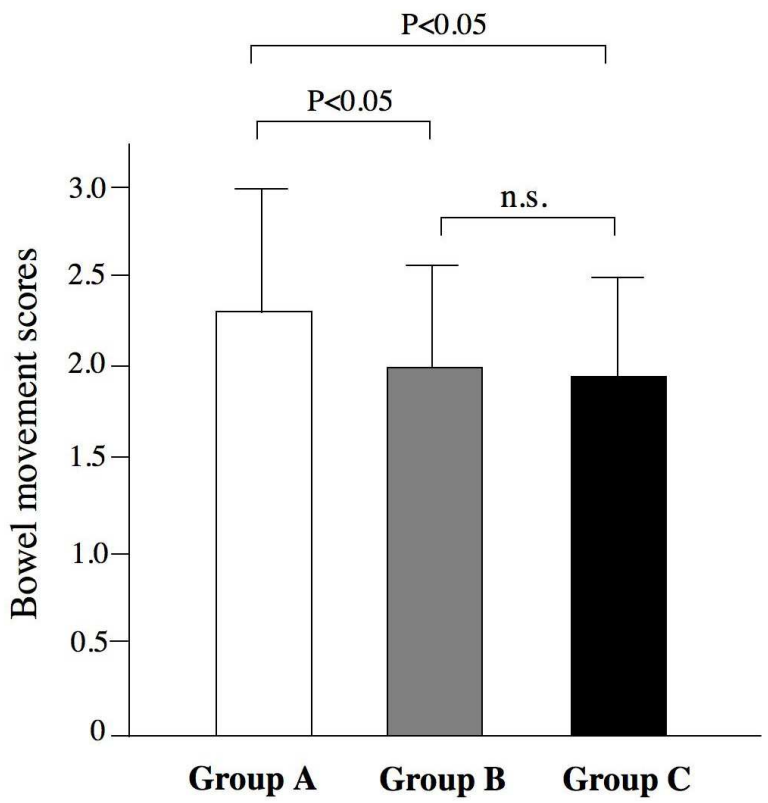

Fig. 4. Relationship between dinner time and bowel movement scores. Bowel movement scores in Group A were significantly higher than those of the other two groups.

\section{Discussion}

For more than a decade, we have been studying the relationship between food habits and quality of life (QOL) in young Japanese women by analyzing the responses to a questionnaire completed by Ashiya College and Kyoto Bunkyo Junior College students. Since the educational and social environment of these populations remained stable throughout this longitudinal survey, we think that the results obtained from these data convincingly represent annual changes in the status of young Japanese women and provide important information.

We previously reported that skipping breakfast is significantly related with poor physical conditions in Japanese female young students (Fujiwara and Nakata, 2010). The present study showed that small population of young students habitually skipped breakfast. Fortunately, this percentage seems to have decreased in recent years (Figure 1). However, it should be noted that accumulating evidence has been suggesting that habits of food intake considerably affects QOL in women. To support this concern, this study clearly showed that students who skip breakfast have a significantly lower frequency of bowel movement compared with that in young women who eat breakfast (Figure 2). This suggests that skipping breakfast induces constipation in young female students. 
In general, food intake especially just after waking up effectively stimulates stomach and induces bowel movement via the parasympathetic nerve pathway. Therefore, it is reasonably speculated that skipping breakfast decreased stimulation to digestive organs in the morning, causing reduction and irregularity of bowel movement. Abnormality in food intake can induce some degree of nutritional defect. Since social activity is relatively high in the morning, skipping breakfast may contribute to a nutritional defect that can affect QOL. However, this study showed that there was no significant difference in BMI among 3 groups (Figure 3). These findings suggest that breakfast skipping had little effects on the total supply of energy for young students and suggested that the positive relationship between breakfast skipping and constipation is due to the disarrangement of the rhythms of automatic nerve systems.

Interestingly, the preliminary study suggested a new proposal that early intake of dinner can improve bowel movement in young female students. Although we cannot exclude the effects of factors accompanying the early intake of dinner, these data notably demonstrated that intake of dinner before 19:00, but not between 19:00 and 21:00, had significant effects on improvement of bowel movement. Considering that intake of dinner between 19:00 and 21:00 has become a common life style practice in modern civilized countries, it is suggested that an intrinsic ideal dietary rhythm for bowel movement in humans differs from our dietary habits.

From the sequential survey conducted in this study, we also found that skipping breakfast is associated with female reproductive disorders such as dysmenorrhea (Fujiwara T, 2003) and proposed that impairment of reproductive function by skipping breakfast may become a trigger for the subsequent onset of gynecologic diseases such as endometriosis in the future (Fujiwara T, 2007). Thus, although the precise mechanisms are still unknown, it is speculated that physiological or pathological conditions of the pelvic organs can be influenced by dietary habits (Fujiwara et al., 2009). Consequently, considering the possibility that constipation influences the environment of pelvic cavity, the relationship between constipation and female genital organic disorders should be examined in the future.

\section{Conclusion}

In conclusion, by conducting a questionnaire survey, this study suggested that early intake of dinner promoted, while skipping breakfast reduced bowel movement in young Japanese female students. These findings support the current concept that dietary habits regulate bowel functions. Since constipation can cause psychological and/or organic disorders, this issue should be re-evaluated from the perspective of dietary rhythm.

\section{References}

Cummings, J.H. (1984) Constipation, dietary fibre and the control of large bowel function. Postgraduate Medical Journal, 60, 811-819.

Fujiwara, T. (2003). Skipping breakfast is associated with dysmenorrhea in young women in Japan. International Journal of Food Science and Nutrition, 54, 505-509. 
Fujiwara, T. (2007). Diet during adolescence is a trigger for subsequent development of dysmenorrhea in young women. International Journal of Food Science and Nutrition, $58,437-444$.

Fujiwara, T., Sato, N., Awaji, H., Sakamoto, H., \& Nakata, R. (2009). Skipping Breakfast Adversely Affects Menstrual Disorders in Young College Students. International Journal of Food Science and Nutrition, 26, 1-9.

Fujiwara, T. \& Nakata R. (2010) Skipping breakfast is associated with reproductive dysfunction in post-adolescent female college students. Appetite, 55, 714-717.

Kunimoto, M., Nishi, M., \& Sasaki K. (1998) The relation between irregular bowel movement and the lifestyle of working women. Hepatogastroenterology, 45, 956-960.

Noble, E.J., Harris, R., Hosie, K.B., Thomas, S. \& Lewis, S.J. (2009) Gum chewing reduces postoperative ileus? A systematic review and meta-analysis. International Journal of Surgery, 7, 100-105.

Whitehead, W.E. (1996) Psychosocial aspects of functional gastrointestinal disorders. Gastroenterology Clinics of North America, 25, 21-34. 


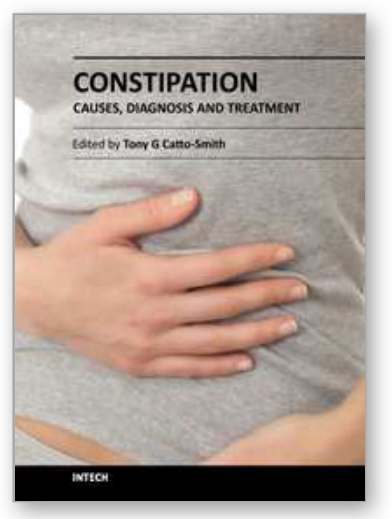

\author{
Constipation - Causes, Diagnosis and Treatment \\ Edited by Dr. Anthony Catto-Smith
}

ISBN 978-953-51-0237-3

Hard cover, 172 pages

Publisher InTech

Published online 07, March, 2012

Published in print edition March, 2012

Constipation is common in both adults and children. Estimates would suggest a median prevalence of around $12-16 \%$ in the general population. While regarded as a minor nuisance in some cases, its consequences can be severe, with a substantial impact on quality of life. Secondary faecal soiling has a profound psychological effect at all ages. This book provides contributions from authors with a range of backgrounds which clarify the pathogenesis, diagnosis, and therapy of constipation for the general population and also for certain high risk groups.

\title{
How to reference
}

In order to correctly reference this scholarly work, feel free to copy and paste the following:

Tomoko Fujiwara (2012). Skipping Breakfast is Associated with Constipation in Post-Adolescent Female College Students in Japan, Constipation - Causes, Diagnosis and Treatment, Dr. Anthony Catto-Smith (Ed.), ISBN: 978-953-51-0237-3, InTech, Available from: http://www.intechopen.com/books/constipation-causesdiagnosis-and-treatment/skipping-breakfast-is-associated-with-constipation-in-post-adolescent-female-collegestudents-in-jap

\section{INTECH}

open science | open minds

\section{InTech Europe}

University Campus STeP Ri

Slavka Krautzeka 83/A

51000 Rijeka, Croatia

Phone: +385 (51) 770447

Fax: +385 (51) 686166

www.intechopen.com

\section{InTech China}

Unit 405, Office Block, Hotel Equatorial Shanghai

No.65, Yan An Road (West), Shanghai, 200040, China

中国上海市延安西路65号上海国际贵都大饭店办公楼405单元

Phone: +86-21-62489820

Fax: $+86-21-62489821$ 
(C) 2012 The Author(s). Licensee IntechOpen. This is an open access article distributed under the terms of the Creative Commons Attribution 3.0 License, which permits unrestricted use, distribution, and reproduction in any medium, provided the original work is properly cited. 